\title{
¿HA SIDO LA REGIÓN CHOROTEGA UN TERRITORIO OLVIDADO? POLÍTICAS DE DESARROLLO SOCIAL Y PRODUCTIVO, 1950-2014
}

\author{
HAS CHOROTEGA REGION BEEN FORGOTTEN BY NATIONAL STATE? \\ POLICIES FOR SOCIAL AND PRODUCTIVE DEVELOPMENT IN \\ CHOROTEGA REGION OF COSTA RICA, 1950-2014
}

\author{
Edgar Eduardo Blanco Obando*
}

\section{RESUMEN}

En este estudio se evidencia una abundante inversión estatal a nivel social y productivo en la región Chorotega, que permitió su plena incorporación al modelo económico nacional, con base en el análisis de trayectoria de numerosos datos de planes de gobierno, literatura científica, anuarios de acciones oficiales concretadas y proyectos gubernamentales concluidos entre 1950 y el 2014. Con esta incorporación, la región ha recibido una significativa inversión en servicios sociales y en infraestructura productiva, convirténdola en uno de los polos económicos más importantes del país. Sin embargo, a pesar de esta situación, no se resolvieron las problemáticas de desempleo, pobreza y desigualdad, que afectan a gran parte de la población guanacasteca.

PALABRAS CLAVE: HISTORIA * DESARROLLO REGIONAL * POLÍTICA ECONÓMICA * ECONOMÍA AGRARIA * TURISMO

\section{ABSTRACT}

The longitudinally analysis about official data and governmental plans and actions for Chorotega region's development, including what governments promised and materialized, has demonstrated the successful region's integration process to national economic and social model in the study period. Thus, this territory had received important public investment in social services and productive infrastructure that built main projects in

1 Este trabajo corresponde a los resultados del proyecto "La Región Chorotega: Políticas de desarrollo y sociales desde el Gobierno Central y desde la Región, 1950-2012. Principales efectos y resultados", inscrito en el Programa Historia Regional y Ambiental Comparada del Centro de Investigaciones Históricas de América Central de la Universidad de Costa Rica, en el 2012, bajo la dirección del Dr. Juan José Marín Hernández.

* Centro de Investigaciones Históricas de América Central, Universidad de Costa Rica, Costa Rica.

edgar.blanco@ucr.ac.cr 
tourism and agriculture areas, but is had been not enough to resolve traditional social problems like unemployment and poverty.

KEYWORDS: HISTORY * REGIONAL DEVELOPMENT * ECONOMIC POLICY * AGRICULTURAL ECONOMICS * TOURISM

\section{INTRODUCCIÓN}

La región Chorotega comprende el territorio de la provincia del Guanacaste, sus 11 cantones (Liberia, Nicoya, Santa Cruz, Bagaces, Carrillo, Cañas, Abangares, Tilarán, Nandayure, La Cruz, Hojancha) junto a los distritos peninsulares de Lepanto, Paquera $y$ Cóbano. Esta región ha presentado un proceso particular de adhesión al modelo de EstadoNación costarricense, debido a que la mayor parte de su actual territorio correspondió en época colonial al Corregimiento o Partido de Nicoya, como parte del Reino de Guatemala junto a las demás provincias centroamericanas, y definió su anexión a lo que actualmente es Costa Rica hasta 1824, tres años posterior a la independencia del istmo.

Para Núñez y Marín (2009), el proceso de inserción del nuevo territorio al modelo económico, político y cultural imperante en Costa Rica para la primera mitad del siglo XIX, se sustentó en el pacto entre las élites regionales y nacionales, que permitió que los recursos naturales y sistemas económicos de la región fueran incorporados al modelo productivo nacional, a cambio de recibir una importante inversión en educación, sanidad y en infraestructura productiva y de servicios.

Para inicios de la segunda mitad del siglo XIX, el concepto de progreso y desarrollo se basaba en un dominio y presencia del Estado a lo largo de todo el país, con capacidad de hacer regir un único sistema ideológico y económico donde la periferia estaba unida al centro económico-político, gracias al impulso del poblamiento en áreas lejanas, construcción de moderna infraestructura productiva, vías de comunicación y la expansión de la educación, junto con el comercio y las actividades económicas que insertaran al país en los principales mercados internacionales (Viales, 2000a).
De este modo, durante la segunda mitad del siglo XIX y una parte del siglo xx, el Estado realizó importantes esfuerzos para promover el crecimiento de las economías de la periferia $y$ asegurar la comunicación entre las regiones con la zona del centro del país, principal polo económico y político nacional ${ }^{2}$; esto principalmente, mediante la construcción de eficientes vías de comunicación y moderna infraestructura productiva, que aseguraran la unión de todo el territorio dentro de un solo modelo económico y político regido por las élites radicadas en el Valle Central (Viales, 2000).

Sin embargo, durante la primera mitad del siglo xx, este accionar fue catalogado como insuficiente por parte de diversos sectores guanacastecos, que percibían en su provincia un atraso importante a nivel de servicios sociales, así como de sistemas viales y productivos, por lo que denunciaron que Guanacaste era una provincia aislada del centro del país y olvidada por el Estado (Alfaro, 2014).

Para sectores intelectuales, la inversión en educación era reducida y existía un severo faltante de escuelas y colegios (Núñez y Marín, 2009). Por su parte, la principal queja de los grandes productores fue la precariedad de las vías de comunicación y el atraso de la infraestructura productiva, que dificultaba el comercio y el desarrollo económico de la región, lo que evidenciaba una enorme diferencia entre la inversión que recibía Guanacaste con respecto a las provincias del Valle Central; situación mantenida prácticamente desde la Anexión (Buska, 2006).

$2 \quad$ El estudio de Zapata y Blanco (2013) muestra las acciones del Estado para incorporar a otra región periférica al modelo económico nacional, por lo que aporta elementos importantes para comprender el actuar del Estado en la región Chorotega dentro del contexto histórico específico de la segunda mitad del siglo xx. 
En esta primera mitad del siglo xx, las vías de comunicación que unían a la región con el resto del país consistían en unos rudimentarios caminos de tierra transitables solo en la estación seca, y en el cabotaje a través del río Tempisque y el golfo de Nicoya, que era la única vía que permitía, a pesar de sus limitantes estructurales, el contacto a lo interno de la región y hacia las poblaciones del Valle Central durante todo el año (Zeledón, 2009).

El cabotaje funcionó como el principal medio de comercio y traslado de personas hacia el Valle Central hasta prácticamente los inicios de la década de 1970, cuando la entrada en pleno funcionamiento de la carretera Interamericana transformó la red vial regional y las comunicaciones con el centro del país. Esta carretera posibilitó la introducción de mejores condiciones para la expansión de la inversión pública en servicios e infraestructura económica y productiva, necesaria para incorporar a la región en el modelo de desarrollo nacional (Núñez y Marín, 2009).

Para Zeledón (2009), la Interamericana además de transformar el sistema vial y productivo guanacasteco, permitió también el control del espacio de la región por parte del Estado, con lo cual, el aislamiento de Guanacaste con respecto al resto del país tendió a reducirse en el tiempo. En este contexto, surge el interés por valorar el tipo de inversión estatal realizada a nivel productivo y social en la región Chorotega, entre los años 1950 y 2014, con el fin de determinar el nivel de incorporación de la región al modelo económico nacional, centrando el análisis sobre lo que el Estado prometió, lo que se ejecutó y los resultados finales obtenidos, valorados desde el grado de satisfacción de las necesidades de la población.

Por su parte, el período definido comprende la superación de los principales obstáculos infraestructurales para la plena incorporación de la región al modelo económico nacional, que se concibe como la expansión de los servicios básicos entre la población y el desarrollo de los sistemas productivos. Dicho análisis se ha realizado mediante la revisión en trayectoria de numerosos documentos: datos de planes de gobierno, literatura científica, anuarios de acciones oficiales concretadas y proyectos gubernamentales concluidos entre 1950 y 2014. 
MAPA 1

POLOS DE DESARROLLO AGRÍCOLA Y TURÍSTICO EN LA REGIÓN CHOROTEGA

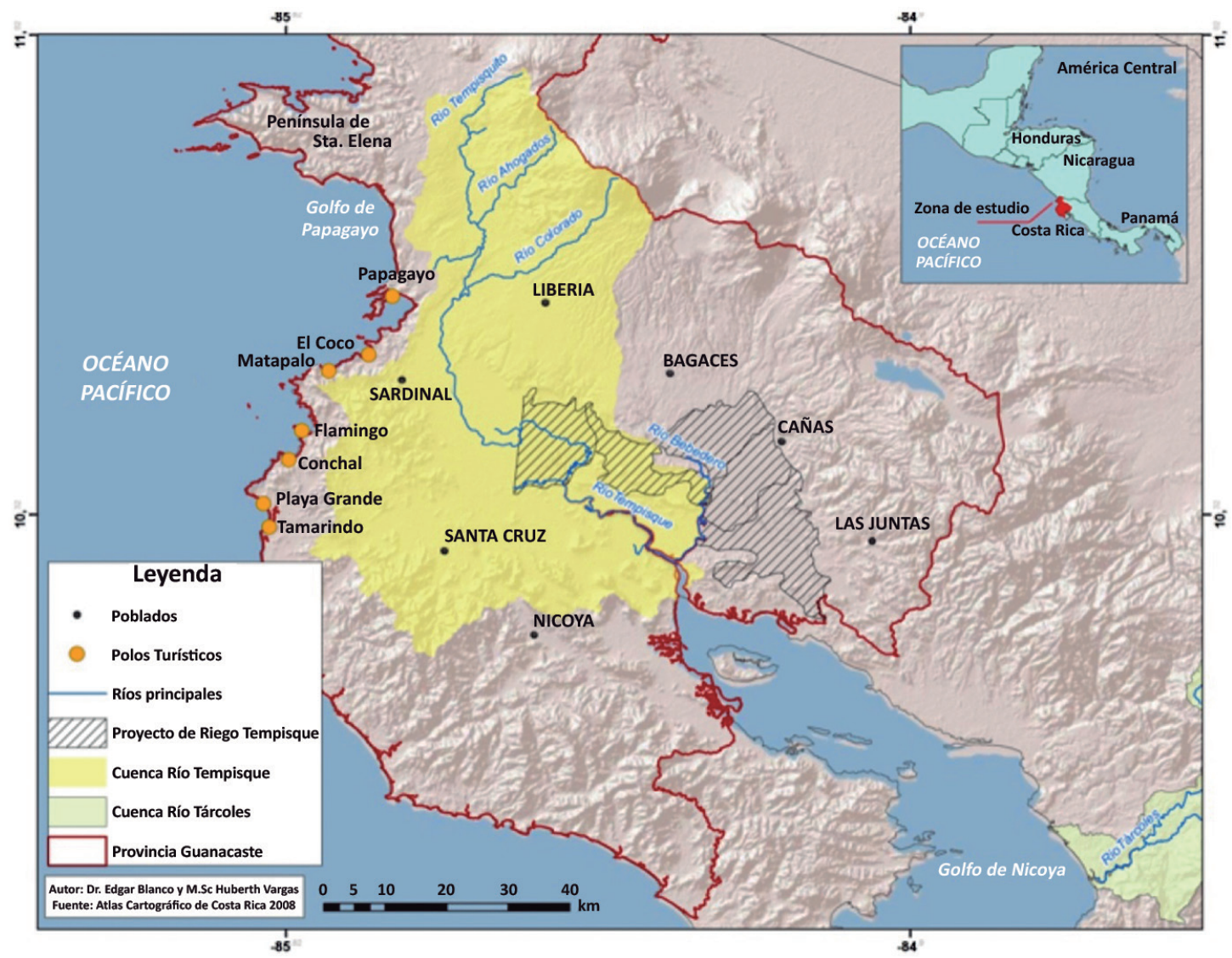

Fuente: Atlas Cartográfico de Costa Rica, p. 10. Instituto Tecnológico de Costa Rica, 2008.

\section{MODELOS ECONÓMICOS INSTAURADOS}

Los modelos económicos implementados en Costa Rica se han dirigido a impulsar el desarrollo del país y solventar las necesidades de la población, con base en las propuestas económicas y productivas construidas por teóricos de los Estados desarrollados, de las agencias de cooperación y de los organismos financieros internacionales como el Banco Mundial (BM), el Fondo Monetario Internacional (FMI), la Organización para la Cooperación y el Desarrollo Económico (OECD, por sus siglas en inglés) o la Comisión Económica para América Latina (CEPAL).
En las primeras décadas de la segunda mitad del siglo xx, la principal preocupación fue la industrialización de los sistemas productivos, por lo que se impulsó el desarrollo del sistema agroexportador, destinando sustanciosos recursos a la modernización de puertos marinos y aéreos, así como a la construcción de carreteras que posibilitaran la comunicación a lo interno de las regiones $y$ de estas con el Valle Central, al igual que a la consolidación de un robusto mercado interno, lograr el cambio tecnológico de las unidades productivas y la cobertura universal de los servicios públicos. Posteriormente, desde mediados del decenio de 1980, la principal estrategia productiva se basó en la inserción de la economía en los mercados mundiales con 
productos novedosos y competitivos, junto con el desarrollo del sector servicios, principalmente turísticos.

\section{TEORÍAS DE LA MODERNIZACIÓN}

Las teorías de la modernización surgieron durante la postguerra, con la propuesta de que los países en desarrollo debían seguir el mismo proceso evolutivo de las naciones desarrolladas, manejando un concepto del desarrollo meramente económico, sustentado en la creación de riqueza y una modernización importante de los sistemas productivos, con el fin de que lograran el crecimiento económico y el incremento del Producto Interno Bruto Nacional (Delgado, 2004).

Así las cosas, para lograr el desarrollo, cada nación tenía que seguir aspectos específicos a nivel económico, social, político y cultural propios de las naciones desarrolladas, con el fin de adquirir conocimientos esenciales y alcanzar el cambio tecnológico y cultural que les permitiera lograr la industrialización (Kay, 2005).

Aunque estas medidas lograron cierto incremento y eficacia en la producción, sus beneficios se concentraron en los grandes empresarios que pudieron asumir la mecanización de sus unidades productivas, provocando la exclusión y endeudamiento de los sectores incapaces de asumir el cambio tecnológico (Delgado, 2004).

\section{MODELO DESARROLLISTA}

El modelo desarrollista rige prácticamente durante las décadas de 1960, 1970 y 1980; se instauró como respuesta a la incapacidad de las teorías de la modernización para resolver los problemas del subdesarro1lo. Este modelo se basó en colocar al Estado como el principal agente rector del desarro$1 l o$, el fomento de la industria local y la sustitución del consumo de bienes importados por los nacionales.

Se impulsó así el desarrollo industrial autóctono a través de la instauración de todo un sistema de subsidios para la producción, la transferencia de información y tecnología, la regulación de los mercados y de la economía por parte del Estado, junto al funcionamiento de numerosos aranceles $y$ acciones proteccionistas contra las importaciones (Bandeira, Atance y Sumpsi, 2004).

De este modo, se instauró en el país el llamado Estado Empresario o Estado Interventor, sustentado en la operación de numerosas empresas públicas dirigidas a regir el desarrollo nacional y asegurar la satisfacción de muchas de las necesidades de la población. La principal muestra de este corporativismo estatal fue la Corporación Costarricense de Desarrollo (CODESA), un enorme consorcio de empresas públicas dedicado al impulso de la producción, la economía y el desarrollo general del país (Rovira, 1987).

Para el desarrollo de la agricultura, definida entonces como el principal sector productivo, se brindó apoyo a la producción de alimentos para el mercado nacional $y$ a las exportaciones, mediante el fomento del cambio tecnológico en las unidades productivas, la compra de semillas mejoradas y una intensiva aplicación de agroquímicos; para así generar los recursos suficientes para sustentar el crecimiento de la industria y de la economía en general (León et ál., 2014).

A pesar de haberse conseguido un crecimiento importante de la producción y de la economía, el sistema intervencionista resultó excesivamente caro, por lo que consumió la mayor parte de los recursos públicos, incluyendo los provenientes de los préstamos externos, lo que llevó al crecimiento desmedido del déficit fiscal $y$ de las deudas interna y externa.

Este desbalance económico a inicios del decenio de 1980, se volvió insostenible a causa en buena parte de la reducción de los ingresos generados dentro del Mercado Común Centroamericano por los conflictos bélicos que se vivían en la región, además del incremento desmedido en los precios mundiales de los hidrocarburos y de las tasas mundiales de interés; esto ocasionó que el país perdiera gran parte de su capacidad productiva y se redujera el PIB, el cual solo entre 1982 y 1983 se contrajo en cerca del 10\% (León, 2012). 


\section{MODELO NEOLIBERAL}

A causa de la inestabilidad económica y el estancamiento del sistema productivo, el país estableció nuevas negociaciones con el Banco Mundial y el Fondo Monetario Internacional, con el fin de obtener recursos externos para reactivar la economía y la producción. Con esto, se logró acceso a nuevos créditos a cambio de la reforma de la política económica y productiva de acuerdo a las nuevas interpretaciones de las teorías económicas clásicas liberales, sustentadas en el desarrollo hacia afuera, la internacionalización de la economía, la liberalización del comercio, la reducción del gasto público, el crecimiento de las exportaciones y la generación de divisas.

A mediados de la década de 1980, el país inició la ejecución del nuevo plan de desarrollo llamado Estrategia de ajuste estructural y promoción de exportaciones, basado en la modernización de la economía y su mayor inserción en los mercados internacionales, con el fin de incrementar la generación de divisas para sanear las finanzas públicas (León et ál., 2014).

Este plan consistió en la instauración de numerosas políticas de índole fiscal, económicas $y$ productivas orientadas a institucionalizar la apertura de los mercados, la reducción del intervencionismo estatal, la contracción del gasto público, el crecimiento de las exportaciones, la atracción de inversión privada y el apoyo a aquellas actividades productivas de mayor dinamismo y rentabilidad. Como resultado de estas medidas, se eliminaron aranceles y el proteccionismo estatal, se redujo el tamaño del Estado, se promovió la inversión privada principalmente transnacional, así como, se asignaron ayudas y estímulos a los grandes exportadores e inversores capaces de competir exitosamente en los mercados internacionales y crear divisas, dedicados principalmente a la exportación de los denominados productos no tradicionales: frutas, flores y otros bienes exóticos de gran demanda en el mercado internacional; $y$ al sector terciario, principalmente al turismo y los servicios financieros (León et ál., 2014).

A partir del decenio de 1990, el apoyo al desarrollo del turismo posicionó al sector como uno de los de mayor crecimiento e importancia, reflejado en su capacidad de crear riqueza. Así, la actividad turística se ha convertido en una de las principales actividades productivas del país, superando en la generación de ingresos a otros productos históricos de exportación como el banano y el café. De 1996 al 2015, el turismo generó \$7373,9 millones en divisas, $\$ 4415,4$ millones más que el banano y $\$ 6211,7$ millones más que el café (ICT, 2008 y 2015).

\section{LOS PROYECTOS PROPUESTOS PARA LA REGIÓN CHOROTEGA}

Entre las décadas de 1950 y de 1960, en los planes de los gobiernos se propuso reiteradamente la ejecución de varios proyectos: la construcción y el mejoramiento de diferentes tramos de la carretera Interamericana y su conexión con los caminos vecinales, con el fin de dinamizar la comunicación al interno de la región y con el Valle Central; el fomento de las cooperativas de electricidad para llevar el servicio a pueblos como Filadelfia, Belén, $\mathrm{Pa}$ lestina, Portegolpe, Bolsón, Ballena, Guardia y Veintisiete de Abril; la construcción de centrales hidroeléctricas en Colorado de Liberia, Filadelfia, Cañas y Tilarán; $y$ centrales térmicas en Liberia, Bagaces, Santa Cruz y Nicoya. Se proyectó también, la construcción de 80 acueductos para el beneficio de 331 comunidades, junto con redes colectoras de aguas negras y su tratamiento en Liberia, Cañas, Santa Cruz, Bagaces, Nicoya, Filadelfia y Tilarán. Para la atención de la salud, se propuso equipar y mejorar los servicios de los hospitales de la región (OPPR, 1966).

Para el desarrollo del sector agropecuario, se propuso el apoyo a la ganadería, el fomento de los cultivos de la caña de azúcar, el algodón, el arroz y la explotación de la madera, tanto para el consumo nacional como para la exportación (OPPR, 1966). Desde la segunda mitad del siglo xx, el incentivo de estas actividades se comprende dentro del contexto de que los cultivos extensivos como arroz y caña de azúcar se desplazaron del Valle Central hacia las regiones periféricas, principalmente al Pacífico Norte y al Pacífico Central, a causa de la ejecución de procesos y paquetes de modernización de las producciones que requerían 
terrenos más planos y climas más cálidos, y de la apertura de vías de comunicación como la carretera Interamericana, que permitieron la incorporación de mayores extensiones de tierras y redujeron los costos del traslado de los productos (Arroyo y León, 2012).

En la década de 1970, los gobiernos idearon diversos proyectos para el crecimiento de las actividades agropecuarias mediante el uso intensivo de recursos naturales, con el fin de obtener cosechas más abundantes y de mejor calidad. Así, se planteó la construcción de los llamados distritos de riego, basados en el uso de las aguas residuales del proyecto hidroeléctrico de Arenal, con el fin de irrigar una extensa superficie de tierras agrícolas.

[...] la posibilidad que ofrece el regadío para cambiar la estructura productiva en la región y para organizar la producción bajo otras bases, constituye un medio sumamente eficaz y de gran impacto para la región y el país, pues solamente en el proyecto de Arenal se irrigarán aproximadamente 100 mil hectáreas, lo que equivale a cerca de un tercio de la actual superficie cultivada. Este proyecto si bien posee un plazo de maduración largo, iniciará la ejecución de su primera etapa a fines de 1976 y ya en 1978 se tendrán las primeras cosechas como fruto del esfuerzo emprendido en el quinquenio (PLN, 1973, p.24).

Se propuso también promover la agroindustria y mejorar la infraestructura agrícola mediante la construcción de una terminal a granel en Punta Morales para exportar azúcar, intensificar la construcción de la carretera entre Cañas y Upala, convertir al aeropuerto Llano Grande de Liberia en un aeródromo de nivel internacional, fomentar la ganadería de carne y de leche, modernizar el sistema de comercialización agrícola junto a los seguros de cosecha y los programas de sanidad agropecuaria (PLN, 1973).

De manera paralela, se planeó impulsar el desarrollo de la industria del turismo con base en el aprovechamiento de los atractivos naturales, principalmente mediante la construcción del Proyecto Turístico de Bahía Culebra, articulado al desarrollo del aeropuerto Llano Grande (OPNPE, 1979).

Para la década de 1980, los proyectos de desarrollo continuaron dentro de las áreas de la agroindustria, el turismo, así como, el mejoramiento y construcción de infraestructura vial $y$ productiva. Durante la primera mitad de esta década, se continuó promocionando el proyecto turístico Bahía Culebra-Golfo de Papagayo, desde la propuesta de construcción de una ley para regir su ejecución. En el campo de la agricultura, las propuestas se centraron en el apoyo al cultivo extensivo de la caña de azúcar y el algodón, diversificar la producción agrícola con la introducción de cultivos como frutas e irrigar 120000 ha en los cantones de Abangares, Cañas, Bagaces, Liberia, Carrillo y Santa Cruz, mediante la ampliación del Proyecto de Riego Arenal-Tempisque (MIDEPLAN, 1982).

En la segunda mitad de la década de 1980 , los proyectos continuaron centrados en el apoyo a los tradicionales sectores productivos primarios, con especial énfasis en la ganadería y la caña de azúcar; también, se planteó la adquisición de nuevas tierras para el Proyecto de Riego Arenal-Tempisque. Con respecto a la infraestructura vial, se prometió continuar con la modernización del aeropuerto de Llano Grande y mejorar los caminos vecinales en distritos alejados como La Cruz (MIDEPLAN, 1987).

En la siguiente década de 1990, se prometieron mayores acciones para poner en funcionamiento el anhelado proyecto turístico del Golfo de Papagayo, ahora mediante un Plan Maestro de Desarrollo para asegurar su funcionamiento. En el tema de infraestructura, se anunció la construcción del puente sobre el río Tempisque, la modernización del Llano Grande y el mejoramiento de la red vial interna en toda la región. Para el Proyecto de Riego ArenalTempisque, se propuso la creación de mayor infraestructura para beneficiar a 100 parceleros de los asentamientos de San Ramón y Playitas en Bagaces, $y$ ampliar en 10000 ha los terrenos bajo irrigación. En el área de la salud, se anunció la mejora de la planta física del hospital de Liberia (MIDEPLAN, 1991, 1994 y 2001). 
En las décadas siguientes, los proyectos se dirigieron mayoritariamente al desarrollo del turismo en toda la región, y en especial a dotar de infraestructura al Polo Turístico de Golfo Papagayo (MIDEPLAN, 2001, 2003, 2007 y 2010).

\section{LOS PROYECTOS CONCRETADOS}

\section{CONSTRUCCIÓN DE VÍAS DE COMUNICACIÓN}

En los inicios de la segunda mitad del siglo xx, la principal obra vial por su magnitud e impacto sobre la incorporación de la región al resto del país fue la carretera Interamericana, cuya construcción correspondió al deseo de los Estados Unidos de contar con una vía panamericana que les permitiera asegurar la supremacía militar en todo el continente. Aunque dicho proyecto se inició en la década de 1940, fue en la de 1960 cuando la carretera se concluyó, pasando a convertirse en la vía de mayor importancia en el país, $y$ arteria articuladora de la red de caminos regionales (Serrano, 1976).

En lo que respecta a la labor directa del Estado en la construcción de obras civiles, fue primero responsabilidad del Ministerio de Transportes (MT), transformado posteriormente en el Ministerio de Obras Públicas y Transportes (МОРт) en la década de 1970. Así, entre 1959 y 1960, el MT construyó y dio mantenimiento a diversas vías locales como la de Santa CruzPlaya Tamarindo, Interamericana-Cañas Dulces de Liberia, Filadelfia-Ballena, Nicoya-Puerto Jesús y Jicaral-Cerro Frío (mT, 1960).

En el decenio de 1970, además de la construcción y mantenimiento de carreteras y puentes, se realizaron obras de mejora de las instalaciones en las terminales aeroportuarias de Liberia y Sámara, con el fin de apoyar la actividad turística en la región (MOPT, 1974 y 1975). En el caso del aeropuerto Llano Grande, el MOPT inició su acondicionamiento como terminal internacional en esta década, labor que culminó hasta inicios del decenio de 1990 (моРт, 1993).

Para la década del 2000, la mayor obra fue la construcción del puente sobre el río Tempisque, donada por el Estado de Taiwán. Esta obra significó la apertura de una vía de comunicación más directa entre el Valle Central con la península de Nicoya, que reduce el viaje en cerca de dos horas, al ser alterna a la ruta por Liberia $y$ Santa Cruz (MOPт, 2001).

\section{INVERSIÓN EN LOS SISTEMAS PRODUCTIVOS}

\section{SECTOR AGRARIO}

Entre las décadas de 1950 y 1980, el principal sector productivo en la región fue el agropecuario, que recibió continuo apoyo e inversión con el fin de asegurar el abastecimiento de alimentos para la población, así como, las materias primas para el creciente sector industrial (León, 2012).

Durante la década de 1950, el principal ente encargado del impulso del sector fue el Ministerio de Agricultura e Industria (MAI), mediante la asignación de recursos, la distribución de tierras y la construcción de infraestructura para la producción. A inicios del decenio, el MAI dedicó importantes recursos a la construcción de obras de riego agrícola, aprovechando las aguas de los ríos de la región.

En la finca Paso Hondo se está actualmente construyendo un canal de derivación con capacidad de 1200 Litros por segundo que tomando las aguas del río Corobicin las conduce hasta la parte más alta de la finca desde donde se comenzará un sistema de distribución para el riego de pastos y de otras cosechas propias de la región; el área que se piensa abarcará la terminación del proyecto total es aproximadamente de 1200 hectáreas y una vez terminado el canal principal se procederá, en un plazo de 3 a 5 años, a distribuir el agua en forma racional sobre la superficie por regar, efectuando este trabajo a razón de 250 a 300 manzanas por año (MAI, 1952, p.166).

De igual forma, el MAI construyó un canal para sacar agua del río Colorado para irrigar un área de 500 manzanas en la finca La Cueva y apoyó en la hacienda El Pelón de la Bajura en la construcción de obras para tomar 
las aguas del río Salto e irrigar 250 manzanas, al igual que la construcción de diques para evitar que el desborde del río inundara las tierras bajas de la finca. A su vez, en la hacienda El Viejo, el Ministerio construyó un canal de 500 metros y una instalación de bombeo en el río Tempisque para regar 200 manzanas. Finalmente, en la hacienda Los Inocentes, se realizaron estudios para construir un canal de 2000 metros de longitud con capacidad de 800 litros por segundo para regar 850 manzanas de pastos y cultivos. Otros latifundios importantes como El Asientillo, El Porvenir y El Pelón de la Altura, también recibieron obras e inversión de parte del MAI (MAI, 1952).

Si bien, estas obras ejecutadas con fondos públicos se realizaron en propiedades de los grandes terratenientes guanacastecos, esto se debió según el MAI, a que presentaban condiciones aptas para la irrigación, según el dictamen de la sección de Ingeniería Rural del Departamento de Servicios Técnicos Especializados (MAI, 1952).

En el decenio de 1960, el MAI es transformado en el Ministerio de Agricultura y Ganadería (MAG), que continuó con las funciones de impulsar y regular el desarrollo del sector primario nacional. Así, el MAG apoyó en la región, el crecimiento de la ganadería extensiva y cultivos como los granos básicos y la caña de azúcar, a través de la realización de estudios y el traslado de información y tecnología a los productores (MAG, 1963, 1964 y 1967).

El impulso al cultivo de la caña de azúcar, produjo en la década de 1970, el asentamiento de algunos de los ingenios más modernos y de mayor tamaño, lo que consolidó a la región Chorotega, junto a la Pacífico Central, como las zonas de mayor desarrollo cañero del país. En el caso del arroz, la entrada en operación de las nuevas vías de comunicación fomentó una mayor incorporación de nuevas tierras de cultivo, las cuales por su calidad de planicie, permitieron la aplicación de tecnología moderna, la introducción de variedades nuevas y la mecanización de la producción (León, 2012).

Por su parte, el interés del Estado de consolidar una estructura tecnológica y productiva para asegurar la autosuficiencia alimentaria, impulsó durante las décadas de 1970 y
1980, el crecimiento y atención de los cultivos de maíz y frijoles, que se consolidaron junto con el arroz y la caña, como los de mayor extensión en Guanacaste (León, 2012).

El crecimiento sostenido del sector agrícola guanacasteco, se aprecia en el uso de la tierra en las fincas entre 1950 y 1984. En dicho período, los terrenos incorporados a las explotaciones agrarias pasaron de 662669 ha a 772031 ha; por su parte, los porcentajes de estos terrenos dedicados a pastos se incremetaron del $40 \%$ al $62,3 \%$; a su vez, las tierras bajo cultivo pasaron del $10,3 \%$ al $16,1 \%$. Por su parte, el tamaño del hato ganadero guanacasteco creció de 233006 cabezas en 1950 a 517654 en 1984, lo que significo el incremento de 284 648 en la cantidad de reses en posesión de los ganaderos guanacastecos (DGEC, 1953 y 1987).

Con respecto al proyecto de riego Arenal-Tempisque, en la década de 1970 se concluyó la primera etapa, que contempló el diseño de los primeros canales junto con un plan para el riego de 85000 ha de terrenos agrícolas (Edelman, 1987). Para la década de 1980, se echó andar la segunda etapa, que incluyó la construcción y el diseño de más canales; así como, la creación del Servicio $\mathrm{Na}$ cional de Aguas Subterráneas, Riego y Avenamiento (SENARA) para administrar el proyecto. Esta etapa se concluyó en 1997, permitiendo el riego de 15900 ha, las que se extendieron posteriormente a 18400 ha y a 27000 ha en el 2012, dedicadas principalmente al cultivo del arroz, frutas como la sandía y el melón, y a pastizales para la ganadería (Programa Estado de la Nación-PEN, 2013).

Si bien, la instauración del riego en la zona ha impulsado el desarrollo de numerosas agroindustrias especializadas en la ganadería y la producción frutera de exportación, los mayores beneficiados han sido los grandes terratenientes guanacastecos, dueños de la mayor cantidad de tierras dentro del distrito de riego. En el año 2000, el 76\% de las fincas eran menores de 10 ha y ocupaban únicamente el $23 \%$ del área bajo riego, mientras que las extensiones mayores de 200 ha eran el $1,7 \%$ del total de fincas, pero cubrían el $48 \%$ del área bajo riego (PEN, 2000). 
SECTOR TURÍSTICO

El principal ente encargado de promover el turismo ha sido el Instituto Costarricense de Turismo (ICT), fundado en 1955 y convertido posteriormente en una institución descentralizada con carácter ministerial (ICT, 1974).

Desde 1950, el desarrollo turístico en Guanacaste puede dividirse en tres fases: 1) predominio del turismo nacional, 2) década de 1980, llegan en mayor cantidad inversiones internacionales en mediana $y$ pequeña escala $y$ 3) a partir del decenio de 1990, predominio de las grandes corporaciones transnacionalizadas, dedicadas al turismo masivo y al comercio de bienes raíces (PEN, 2000).

Si bien, desde el ICT el Estado ha mantenido un apoyo continuo al crecimiento del turismo en la región, que se incrementó considerablemente a partir de 1990 dentro del contexto de la reconversión productiva neoliberal, el mayor proyecto ha sido la construcción del polo turístico en Bahía Culebra, llamado Proyecto Turístico Golfo de Papagayo, que desde la década de 1970 ha mantenido su ejecución como una política de Estado.

Para el inicio de dicho proyecto, el Estado negoció un préstamo de $\$ 370000$ con el Banco Interamericano de Integración Económica (BCIE) para financiar los estudios de factibilidad técnica y económica, así como para la elaboración del plan maestro de construcción (ICT, 1974). En 1982, se erigió la Ley de Creación del Proyecto Turístico de Papagayo (nro. 6758), que estableció las normas para la ejecución de las acciones correspondientes a la construcción de dicho polo turístico (Asamblea Legislativa de Costa Rica, 1982).

Durante las décadas de 1980 y 1990, el ICT coordinó la construcción de infraestructura de servicios básicos con entidades gubernamentales como Acueductos y Alcantarillados (AYA), el Instituto Costarricense de Electricidad (ICE) $y$ el Ministerio de Obras Públicas y Transportes (МОРТ); y realizó acciones para lograr la expropiación de terrenos para asentar a los concesionarios. Para mediados de la década de 1980, el ICT llegó a controlar 1006 ha en Bahía Culebra y más de 5841 metros cuadrados en la zona marítimo terrestre (ICT, 1985).
Al inicio de la década de 1990, inician su operación las primeras empresas turísticas en el Proyecto Turístico Golfo de Papagayo, con apoyo del ICT $y$ del Estado para satisfacer las necesidades de agua, electricidad, construcción y asfaltado de caminos y concesión de tierras (ICT, 2001). Para el 2008, ya funcionaban 9 empresas hoteleras transnacionales, con un total de 1130 habitaciones, el 5,9\% del total de las habitaciones turísticas a nivel nacional (ICT, 2008).

En general, el importante desarrollo turístico alcanzado en la región Chorotega, se aprecia en la cantidad de empresas de hospedaje en operación, junto a la totalidad de habitaciones disponibles para los turistas, que componen una de las ofertas más amplias del país.

$\mathrm{Al}$ inicio de la década de 1990, Guanacaste contó con 547 habitaciones con declaratoria turística en operación, el 27,3\% del total nacional, que la situó en el tercer lugar entre las provincias con mayor cantidad de estas habitaciones, situándose por debajo de Puntarenas (617) y de San José (618) (ICT, 1991).

Para el año 2003, el número de habitaciones turísticas pasó a 3760, el 24,0\% del total nacional. En dicho año, Guanacaste se situó como la segunda provincia con mayor número de estas habitaciones, por debajo de San José (4536) pero superando a Puntarenas (3509) (ICT, 2003).

En el año 2014, Guanacaste contó con 6818 habitaciones turísticas en oferta, el 30,6\% del total nacional; esto la posisionó como la provincia con mayor número de estas habitaciones en el país, por encima de San José (6050) $y$ de Puntarenas (4351) (IcT, 2014).

Tomando en cuenta el número de empresas de hospedaje, en el 2003 se ubicaron 108 en Guanacaste, el 24,8\% del total nacional, por lo que esta provincia fue la del mayor número de empresas de este tipo, superando a Puntarenas (107) y a San José (102) (ICT, 2003).

En el año 2014, la cantidad de empresas de hospedaje en Guanacaste se redujo a 102, el $21,9 \%$ nacional, esto colocó a la provincia como la tercera en cuanto al número de estas empresas, por debajo de Puntarenas (114) y de San José (115) (ICT, 2015). 
Esta situación de mayor cantidad de habitaciones turísticas en servicio, junto a la tercera mayor cantidad de empresas de hospedaje, se debe a la presencia de un turismo de tipo masivo, que se sustenta en el asentamiento de complejos hoteleros grandes, con centenares de habitaciones.

\section{INVERSIÓN PARA EL BIENESTAR SOCIAL}

SALUD

Con respecto a la inversión estatal en el campo de la salud, la Caja Costarricense del Seguro Social (cCSs) como la principal institución que presta los servicios de atención curativa, ha fundado o modernizado numerosas clínicas ubicadas estratégicamente a lo largo del territorio Chorotega, al igual que los hospitales Enrique Baltodano Briceño en Liberia y La Anexión en Nicoya (Botey, 2013), junto al hospital de Upala en Alajuela, que atiende a poblaciones guanacastecas cercanas.

Según datos de la ccss sobre los principales servicios en atención curativa prestados: consultas médicas, exámenes de laboratorio $y$ medicamentos despachados; se evidencia una significativa inversión para la satisfacción de estas necesidades. El número de consultas médicas entre 1970 y el 2010 se incrementó de 109 497 (6,1\% del país) a 730973 (16\% del país) (cCss, 1970 y 2010).

Por su parte, la cantidad de medicamentos despachados entre 1960 y el 2010 se incrementó de 41499 , el 5,5\% del total de del país, a 5700359 , el 7,2\% del total nacional (ccss, 1960 y 2010). A su vez, la cantidad de exámenes de laboratorio realizados entre 1960 y el 2010, también se incrementó de manera significativa, al pasar de 4569 (5,5\% del total nacional) a 5375782 (el 7,8\% del país) (ccss, 1960 y 2010).

\section{EDUCACIÓN}

El Ministerio de Educación Pública (MEP), principal encargado de regular la educación en el país, ha dirigido constantes recursos para atender las necesidades educativas de la población guanacasteca. En 1950, en Guanacaste funcionaban 204 escuelas, la mayor cantidad entre las provincias periféricas, y la tercera mayor cantidad de estos centros educativos del país, solo por debajo de las provincias metropolitanas de Alajuela y San José.

TABLA 1

CANTIDAD DE ESCUELAS PRIMARIAS POR PROVINCIA EN 1950

\begin{tabular}{ccc}
\hline PROVINCIA & $\begin{array}{c}\text { CANTIDAD } \\
\text { DE ESCUELAS }\end{array}$ & $\%$ \\
\hline San José & 248 & 25,2 \\
Alajuela & 231 & 23,5 \\
Cartago & 113 & 11,5 \\
Heredia & 51 & 5,1 \\
Guanacaste & 204 & 20,7 \\
Puntarenas & 107 & 11 \\
Limón & 27 & 3 \\
\hline TOTAL & 981 & 100 \\
\hline
\end{tabular}

Fuente: Elaboración propia con base en Memoria Anual del MEP, 1950.

Para finales de esta década, el trabajo conjunto del MEP y el Departamento de Arquitectura y Construcción del MT, logró la construcción de aulas en las siguientes instituciones educativas a lo largo de la región: Liceo de Tilarán, Liceo de Nicoya, Kindergarten de Liberia, Escuela de Puerto Humo y Escuela Bernabela de Santa Cruz (mT, 1960).

Este mismo trabajo conjunto interministerial permitió entre 1962 y 1966, la construcción de 173 aulas, la mayor cantidad en las provincias periféricas, $y$ la tercera mayor cantidad a nivel nacional, solo por debajo de San José y Alajuela. 
TABLA 2

NÚMERO DE AULAS ESCOLARES CONSTRUIDAS POR EL MINISTERIO DE TRANSPORTES EN COSTA RICA POR PROVINCIA 1962-1966

\begin{tabular}{ccc}
\hline \multirow{2}{*}{ PROVINCIA } & NÚMERO & $\%$ \\
\hline SEn José & 456 & 41,4 \\
Alajuela & 180 & 16,2 \\
Cartago & 137 & 12,3 \\
Heredia & 108 & 9,7 \\
Guanacaste & 173 & 15,5 \\
Limón & 55 & 4,9 \\
\hline TOTAL & 1109 & 100 \\
\hline
\end{tabular}

Fuente: Elaboración propia con base en datos del MT, 1966.

A su vez, el MEP contrató un número importante de maestros para abastecer la inversión en escuelas. En 1951, se nombraron 558 educadores en Guanacaste, la mayor cantidad entre las provincias periféricas y la cuarta mayor del país, después de Cartago, Alajuela y San José.

TABLA 3

TOTAL DE PERSONAL DOCENTE DE PRIMARIA POR PROVINCIA

1951

\begin{tabular}{ccc}
\hline PROVINCIA & $\begin{array}{c}\text { CANTIDAD DE MAESTROS } \\
\text { Y MAESTRAS }\end{array}$ & $\%$ \\
\hline San José & 1812 & 38,4 \\
Alajuela & 862 & 18,3 \\
Cartago & 609 & 12,8 \\
Heredia & 438 & 9,4 \\
Guanacaste & 558 & 11,8 \\
Puntarenas & 320 & 6,7 \\
Limón & 123 & 2,6 \\
\hline TOTAL & 4722 & 100 \\
\hline
\end{tabular}

Fuente: Elaboración propia con base en Memoria Anual del MEP, 1951.

Con respecto a la secundaria, en 1963, Guanacaste fue la provincia periférica con mayor cantidad de estas instituciones, presentando cifras incluso similares a las provincias vallecentralinas de Heredia y Cartago.
TABLA 4

NÚMERO TOTAL DE COLEGIOS POR PROVINCIA 1963

\begin{tabular}{ccc}
\hline PROVINCIA & CANTIDAD DE COLEGIOS & $\%$ \\
\hline San José & 40 & 52,7 \\
Alajuela & 15 & 19,8 \\
Cartago & 7 & 9,3 \\
Heredia & 5 & 6,5 \\
Guanacaste & 5 & 6,5 \\
Puntarenas & 2 & 2,6 \\
Limón & 2 & 2,6 \\
\hline TOTAL & 76 & 100 \\
\hline
\end{tabular}

Fuente: Elaboración propia con base en Memoria Anual del MEP, 1963.

Por su parte, el nombramiento total de docentes de primaria y secundaria en 1983 para la región Chorotega fue de 889 , la quinta mayor cantidad de nombramientos a nivel nacional $y$ el 11\% del país.

TABLA 5

NOMBRAMIENTO DE PERSONAL DOCENTE EN

GENERAL TRAMITADOS POR DIRECCIONES REGIONALES SEGÚN REGIÓN DEL PAÍS 1983

\begin{tabular}{ccc}
\hline REGIÓN & CANTIDAD DE PERSONAL & $\%$ \\
ACADÉMICA & 2502 & 30,4 \\
\hline Central & 516 & 6,3 \\
Oriental & 1567 & 19 \\
Occidental & 889 & 11 \\
Chorotega & 1201 & 14,5 \\
Brunca & 951 & 11,5 \\
Atlántica & 602 & 7,3 \\
Norte & 8228 & 100 \\
\hline TOTAL & &
\end{tabular}

Fuente: Elaboración propia con base en Memoria Anual del MEP, 1982-1986.

\section{VIVIENDA}

La asignación de viviendas se ha consolidado en el país como labor de tres instituciones: el Instituto Nacional de Vivienda y Urbanismo (INVU), el Ministerio de Vivienda y Asentamientos Humanos (MIVAH) y el Banco Hipotecario de la Vivienda (BANHVI); que asignan soluciones a gente necesitada, al igual que créditos y bonos 
para adquirir o mejorar las casas. El INVU, creado en la década de 1950, fue la primera institución en atender exclusivamente la necesidad habitacional de la población; posteriormente se crearon el MIVAH y el BANHVI en la década de 1980. Entre 1955 y 1985, el INVU asignó 29174 viviendas, de las cuales 1202 se repartieron en Guanacaste, la segunda menor cantidad después de Cartago.

TABLA 6

NÚMERO DE VIVIENDAS CONSTRUIDAS POR EL INVU POR PROVINCIA

ENTRE 1955 Y 1985

\begin{tabular}{ccc}
\hline PROVINCIA & $\begin{array}{c}\text { NRO. DE CASAS } \\
\text { CONSTRUIDAS }\end{array}$ & $\begin{array}{c}\text { \% DE CASAS } \\
\text { CONSTRUIDAS }\end{array}$ \\
\hline San José & 19580 & 67,1 \\
Alajuela & 1949 & 6,6 \\
Cartago & 881 & 3,0 \\
Heredia & 1275 & 4,3 \\
Guanacaste & 1202 & 4,1 \\
Puntarenas & 3054 & 10,4 \\
Limón & 1233 & 4,5 \\
\hline TOTAL & 29174 & 100 \\
\hline
\end{tabular}

Fuente: Elaboración propia con base en Memoria Anual del InvU,1985.

Con respecto a los bonos asignados por el INVU en el 2001 para la compra, reparación o ampliación de vivienda, la segunda mayor cantidad (295) se asignó en Guanacaste, por debajo solo de San José (464).

TABLA 7

ASIGNACIÓN DE BONOS DEL INVU PARA LA VIVIENDA POR PROVINCIA 2001

\begin{tabular}{ccc}
\hline PROVINCIA & NRO. DE BONOS & $\%$ \\
\hline San José & 464 & 31,8 \\
Alajuela & 284 & 19,5 \\
Cartago & 149 & 10,2 \\
Heredia & 47 & 3,2 \\
Guanacaste & 295 & 20,2 \\
Puntarenas & 135 & 9,2 \\
Limón & 86 & 5,9 \\
\hline TOTAL & 1460 & 100 \\
\hline
\end{tabular}

Fuente: Elaboración propia con base en Memoria Anual del INVU, 2001-2002.
Por su parte, tomando los créditos otorgados por el BANHVI en 1988, Guanacaste recibió la cuarta mayor cantidad nacional (1493), pero la mayor entre las provincias periféricas, muy por encima de Puntarenas (814) y Limón (613).

TABLA 8

CANTIDAD DE CRÉDITOS PARA VIVIENDAS OTORGADOS POR PROVINCIA POR EL BANHVI 1988

\begin{tabular}{ccc}
\hline Provincia & $\begin{array}{c}\text { NRO. DE CRÉDITOS } \\
\text { OTORGADOS }\end{array}$ & $\%$ \\
\hline San José & 4003 & 34,3 \\
Alajuela & 2466 & 20,9 \\
Cartago & 1499 & 12,7 \\
Heredia & 880 & 7,4 \\
Guanacaste & 1493 & 12,6 \\
Puntarenas & 814 & 6,9 \\
Limón & 613 & 5,2 \\
\hline TOTAL & 11768 & 100 \\
\hline
\end{tabular}

Fuente: Elaboración propia con base en Memoria del BANHVI, 1988.

Para 1996, del total de créditos formalizados por el BANHVI, Guanacaste (2100) obtuvo la cuarta mayor cantidad nacional, pero la primera entre las provincias de la periferia.

$$
\begin{gathered}
\text { TABLA } 9 \\
\text { CRÉDITOS FORMALIZADOS POR EL BANHVI } \\
\text { SEGÚN PROVINCIA } \\
1996
\end{gathered}
$$

\begin{tabular}{ccc}
\hline PROVINCIA & NRO. DE CASOS & $\%$ \\
\hline San José & 4370 & 25 \\
Alajuela & 4070 & 23 \\
Cartago & 3153 & 18 \\
Heredia & 803 & 5 \\
Guanacaste & 2100 & 12 \\
Puntarenas & 1525 & 9 \\
Limón & 1402 & 8 \\
\hline TOTAL & 17423 & 100 \\
\hline
\end{tabular}

Fuente: Elaboración propia con base en Memoria del BANHVI, 1996.

Finalmente, con respecto a los bonos pagados por el MIVAH en el 2004, Guanacaste (2772) fue la tercera provincia del país con mayor beneficio recibido, por debajo solo de San José (2775) y Alajuela (4544). 
TABLA 10

BONOS PAGADOS POR EL MIVAH SEGÚN PROVINCIA 2004

\begin{tabular}{ccc}
\hline PROVINCIA & $\begin{array}{c}\text { NRO. DE BONOS } \\
\text { PAGADOS }\end{array}$ & $\%$ \\
\hline San José & 2775 & 16,6 \\
Alajuela & 4544 & 26,4 \\
Cartago & 1862 & 10,8 \\
Heredia & 960 & 5,5 \\
Guanacaste & 2772 & 16,1 \\
Puntarenas & 2385 & 13,8 \\
Limón & 1867 & 10,8 \\
\hline TOTAL & 17165 & 100 \\
\hline
\end{tabular}

Fuente: Elaboración propia con base en Memoria Anual del MIVAH, 2002-2004.

\section{CONDICIONES DE VIDA DE LA POBLACIÓN}

\section{DESEMPLEO}

Entre 1990 y 2013, las tasas de desempleo abierto (población desocupada con respecto a la fuerza de trabajo) en los hogares de la región, se mantuvieron superiores a las nacionales, con excepción solamente de los años 1995 y 2005. En términos generales, entre 1990 y el 2013, la tasa de desempleo en la región se incrementó en 5,4 puntos porcentuales, superando al crecimiento de la tasa nacional que fue de 3,9\%.
TABLA 11

TASA DE DESEMPLEO ABIERTO

EN LOS HOGARES DE LA

REGIÓN CHOROTEGA

Y EN EL CONTEXTO NACIONAL

1990-2013

\begin{tabular}{ccc}
\hline AÑO & REGIÓN CHOROTEGA & PAÍS \\
\hline 1990 & 5,8 & 4,6 \\
1995 & 4,5 & 5,2 \\
2000 & 5,7 & 5,2 \\
2005 & 5,8 & 6,6 \\
2010 & 9,6 & 7,3 \\
2012 & 11,4 & 7,8 \\
2013 & 11,2 & 8,5 \\
\hline
\end{tabular}

Fuente: Elaboración propia con base en DGEc, 1990 y 1995, e INEC, 2000, 2005, 2010, 2012 y 2013.

\section{POBREZA}

La pobreza en los hogares guanacastecos, entendida como la presencia de niveles de vida o de bienestar inaceptables en relación con la imposibilidad de satisfacer las necesidades básicas, entre 1987 y 1996 se mantuvo entre el 46,2 y el 34,6\%; mientras que la pobreza extrema mostró porcentajes bastante elevados, entre el $21,4 \%$ y el $13,2 \%$. Durante todo el lapso, los porcentajes de pobreza convencional y extrema en la región fueron muy superiores a los del país.

TABLA 12

PORCENTAJE DE HOGARES EN CONDICIÓN DE POBREZA Y POBREZA EXTREMA EN LA REGIÓN CHOROTEGA Y EN EL CONTEXTO NACIONAL 1987-1996

\begin{tabular}{ccccc}
\hline AÑO & $\begin{array}{c}\text { REGIÓN CHOROTEGA } \\
\text { POBREZA }\end{array}$ & $\begin{array}{c}\text { POBREZA } \\
\text { EXTREMA }\end{array}$ & $\begin{array}{c}\text { PAÍS } \\
\text { POBREZA }\end{array}$ & $\begin{array}{r}\text { POBREZA } \\
\text { EXTREMA }\end{array}$ \\
\hline 1987 & 46,2 & 21,4 & 29,0 & 9,0 \\
1995 & 35,2 & 13,6 & 20,3 & 6,2 \\
1996 & 34,6 & 13,2 & 21,5 & 6,8 \\
\hline
\end{tabular}

Fuente: $\quad$ Elaboración propia con base en DGEC, 1987 y 1995, e INEc, 1996.

Posteriormente, entre el 2005 y el 2013, la pobreza en la región se mantuvo también en porcentajes bastante superiores al país, sin mostrar un descenso importante; mientras que la pobreza extrema mostró un leve aumento y se mantuvo casi duplicando a la nacional. 
TABLA 13

PORCENTAJE DE HOGARES EN POBREZA Y POBREZA EXTREMA

EN LA REGIÓN CHOROTEGA Y EN EL CONTEXTO NACIONAL

2005-2015

\begin{tabular}{ccccc}
\hline AÑO & $\begin{array}{c}\text { REGIÓN CHOROTEGA } \\
\text { POBREZA }\end{array}$ & $\begin{array}{c}\text { POBREZA } \\
\text { EXTREMA }\end{array}$ & $\begin{array}{c}\text { PAÍS } \\
\text { POBREZA }\end{array}$ & $\begin{array}{c}\text { POBREZA } \\
\text { EXTREMA }\end{array}$ \\
\hline 2005 & 34,3 & 11,8 & 23,8 & 6,0 \\
2010 & 32,6 & 11,2 & 21,3 & 6,0 \\
2012 & 34,5 & 12,6 & 20,6 & 6,3 \\
2013 & 34,1 & 12,6 & 20,7 & 6,4 \\
\hline
\end{tabular}

Fuente: Elaboración propia con base en INEc, 2005, 2010, 2012 y 2013.

\section{DESIGUALDAD}

La desigualdad se explica desde la concentración del ingreso y se mide con el Coeficiente de Gini, con una escala ascendente de 0 (igualdad absoluta) a 1 (máxima desigualdad). Entre el 2000 y el 2013, en la región Chorotega permanecieron niveles medios $y$ altos de desigualdad, similares a los presentados por el país.

TABLA 14

COEFICIENTE DE GINI PARA LA REGIÓN CHOROTEGA Y COSTA RICA 2000-2013

\begin{tabular}{ccc}
\hline AÑO & $\begin{array}{c}\text { REGIÓN } \\
\text { CHOROTEGA }\end{array}$ & COSTA RICA \\
\hline 2000 & 0,454 & 0,413 \\
2005 & 0,396 & 0,407 \\
2010 & 0,505 & 0,535 \\
2012 & 0,513 & 0,521 \\
2013 & 0,520 & 0,508 \\
\hline
\end{tabular}

Fuente: $\quad$ Elaboración propia con base en INEc, 2013.

A pesar de que la desigualdad creció y se mantuvo en niveles elevados en ambas poblaciones, en la región Chorotega el incremento fue relativamente inferior $(0,066)$ al nacional $(0,095)$. Es importante señalar que, a causa de que la información necesaria para establecer la desigualdad proviene de las encuestas de ingresos, en donde los que más tienen suelen declarar la presencia de ganancias inferiores, es muy probable que la desigualdad en ambas poblaciones (regional y nacional) sea incluso mayor a la oficialmente establecida.

\section{CONCLUSIONES}

Durante el período de estudio, la región Chorotega recibió continua y sustanciosa inversión estatal para el desarrollo de los sistemas productivos, construcción y mantenimiento de los sistemas viales, así como para la expansión de los servicios entre la población, mediante la aplicación de políticas de desarrollo diseñadas con base en el modelo económico imperante.

En el período de modernizacióndesarrollista, el Estado impulsó la expansión de la cobertura de los servicios públicos entre la población guanacasteca, junto con el desarrollo y la modernización del sistema agroproductivo, logrando el crecimiento sigificativo de la ganadería y de los cultivos de granos básicos y de caña de azúcar. Posteriormente, en el período neoliberal, en Guanacaste se consolidó el mayor polo turístico del país, un importante y moderno sector agrícola dedicado a la exportación y el funcionamiento de un aeropuerto internacional, lo que insertó plenamente la economía regional en el mercado internacional.

De este modo, es posible afirmar que el Estado costarricense logró la plena incorporación de la región Chorotega al modelo económico nacional, mediante una constante inversión en servicios, incluso superior en algunos rubros a la recibida por las otras provincias periféricas 
(Puntarenas y Limón), junto con el desarrollo de los sistemas productivos más dinámicos y rentables. Sin embargo, no se alcanzó a resolver las problemáticas de pobreza, desempleo $y$ desigualdad, que afectan sensiblemente a la población guanacasteca de manera constante en el tiempo.

La explicación de esta situación debe buscarse en el análisis histórico de situaciones estructurales presentes en los procesos de desarrollo, como las características de los modelos productivos aplicados y su relación con la distribución y concentración de la riqueza, la influencia de las élites nacionales y regionales en el planeamiento y ejecución de las políticas de desarrollo, el comportamiento de las entidades gubernamentales nacionales y regionales, el actuar de los partidos políticos, la intromisión de intereses económicos y políticos en el aparato público y el papel desempeñado por la sociedad civil, como actor vigilante de su proceso de desarrollo, seleccionador y fiscalizador de sus gobernantes ${ }^{3}$.

$3 \quad$ Para obtener mayor información sobre los resultados en el contexto social de las políticas económicas aplicadas en Guanacaste, especialmente sobre el por qué el crecimiento económico y productivo no se ha traducido en mejoras significativas en los niveles de vida de la población guanacaste$\mathrm{ca}$, se recomienda la consulta de los siguientes trabajos, donde el autor alcanza una explicación para esta situación: "Medio ambiente y desarrollo: efectos $y$ resultados de las actividades productivas $y$ la legislación ambiental sobre la naturaleza y las condiciones de vida de la población, en la región Chorotega de Costa Rica. 1990-2015”. Diálogos, 17 (1), pp. 3-30. San José, Costa Rica: CIHAC-Universidad de Costa Rica, enero-junio, 2016. "Desarrollo sustentable: ¿Mayor disfrute del medio ambiente $y$ mejores condiciones de vida para las poblaciones locales? Análisis de la región Chorotega en Costa Rica, 1990-2013". Perspectivas Rurales, 26, pp 59-70. Heredia, Costa Rica: Universidad Nacional, 2015. "Desarrollo Rural Territorial: ¿El mejor recurso para resolver las problemáticas de las poblaciones rurales costarricenses? Un análisis del período 1990-2014". Historia de las desigualdades sociales en América Central. Una visión interdisciplinaria siglos XVIII-XXI. En R. Viales y D. Díaz (ed.), pp 158-172. San José, Costa Rica: CIHAC-Universidad de Costa Rica, 2015. "Impacto social de la modernización de los sistemas viales $y$ productivos en la región Chorotega de Costa

\section{REFERENCIAS}

Alfaro, E. (febrero-agosto, 2014). El discurso en la invensión de la Fiesta Nacional de la anexión del partido de Nicoya a Costa Rica.1940-1974. Diálogos, 15(1), 37-75.

Arroyo, N. y León, J. (2012). La modernización agrícola: un proceso de largo plazo visto a través de las experiencias de la caña de azúcar y el arroz. Reflexiones, Jornadas de Investigación Interdisciplinarias.

Asamblea Legislativa de Costa Rica. (1982). Ley de Creación del Proyecto Turístico de Papagayo. San José, Costa Rica: Poder Legislativo.

Banco Hipotecario de la Vivienda (BAHVI). (1988). Memoria Anual 1988. San José, Costa Rica: BAHVI.

Banco Hipotecario de la Vivienda (BAHVI). (1996). Memoria Anual 1996. San José, Costa Rica: BAHVI.

Banderira, P.; Atance, I.; Sumpsi, J. M. (2004). Las políticas de desarrollo rural en América Latina: requerimientos de un nuevo enfoque. Cuadernos de Desarrollo Rural, 51. Bogotá, Colombia: Universidad Javeriana.

Botey, A. (2013). Los actores sociales y la construcción de las politicas de salud del Estado Liberal en Costa Rica 18501949. (Tesis de graduación, Doctorado en Historia). Sistema de Estudios de Postgrado, Universidad de Costa Rica. San José, Costa Rica.

Buska, S. (enero-diciembre, 2006). Guanacaste: El Surgimiento de un Discurso Regionalista, 1900-1926. Revista Historia, 53-54, 143-169.

Caja Costarricense de Seguro Social (ccss). (1960). Memoria Anual 1959-1960. San José, Costa Rica: ccss.

Rica. 1950-2013". Revista de Ciencias Sociales, 153, pp 13-26. San José, Costa Rica: Universidad de Costa Rica, 2016. "¿Testimonios de un despojo? Desarrollo turístico en Guanacaste y sus impactos a nivel social y ambiental, 1990-2016". Revista de Ciencias Sociales, 155, pp 13-27. San José, Costa Rica: Universidad de Costa Rica, 2017. 
Caja Costarricense de Seguro Social (ccss). (1970). Memoria Anual 1970. San José, Costa Rica: ccss.

Caja Costarricense de Seguro Social (ccss). (2010). Indicadores por cantón 2009. San José, Costa Rica: ccss.

Delgado, M. (2004). La Política rural europea en la encrucijada. MAPA, Serie ESTUDIOS. Madrid, España.

Dirección General de Estadísticas y Censos (DGEC). (1953). Censo Agropecuario 1950. San José, Costa Rica: Ministerio de Economía y Hacienda.

Dirección General de Estadísticas y Censos (DGEC). (1987). Censo Agrícola 1984. San José, Costa Rica: Ministerio de Economía, Industria y Comercio.

Dirección General de Estadísticas y Censos (DGEC). (1990). Encuesta de Hogares y Propósitos Múltiples. Módulo de Empleo, 1990. San José, Costa Rica: Ministerio de Economía, Industria y Comercio.

Dirección General de Estadísticas y Censos (DGEC). (1995). Encuesta de Hogares y propósitos Múltiples. Módulo de Empleo, 1995. San José, Costa Rica: Ministerio de Economía, Industria y Comercio.

Edelman, M. (1987). El Distrito de Riego de Guanacaste (Costa Rica) y la Política del Agua. Anuario de Estudios Centroamericanos, 3(1).

Instituto Costarricense de Turismo (ICT). (1974). Memoria Anual 1973. San José, Costa Rica.

Instituto Costarricense de Turismo (ICT). (1985). Memoria Anual 1985. San José, Costa Rica.

Instituto Costarricense de Turismo (ICT). (1991). Memoria Anual 1991. San José, Costa Rica.

Instituto Costarricense de Turismo (ICT). (2001). Memoria Anual 2001. San José, Costa Rica.

Instituto Costarricense de Turismo (ICT). (2003). Memoria Anual 2003. San José, Costa Rica.

Instituto Costarricense de Turismo (ICT). (2008). Memoria Anual 2008. San José, Costa Rica.
Instituto Costarricense de Turismo (ICT). (2014). Memoria Anual 2014. San José, Costa Rica.

Instituto Costarricense de Turismo (ICT). (2015). Anuario Estadístico. San José, Costa Rica.

Instituto Nacional de Estadísticas y Censos (INEC). (1996). Encuesta de Hogares y Propósitos Múltiples. San José, Costa Rica.

Instituto Nacional de Estadísticas y Censos (INEC). (2000). Encuesta de Hogares y Propósitos Múltiples. San José, Costa Rica.

Instituto Nacional de Estadísticas y Censos (INEc). (2005). Encuesta de Hogares y Propósitos Múltiples. San José, Costa Rica.

Instituto Nacional de Estadísticas y Censos (INEC). (2010). Encuesta Nacional de Hogares. San José, Costa Rica.

Instituto Nacional de Estadísticas y Censos (INEC). (2012). Encuesta de Hogares y propósitos Múltiples. San José, Costa Rica.

Instituto Nacional de Estadísticas y Censos (INEC). (2013). Encuesta de Hogares y propósitos Múltiples. San José, Costa Rica.

Instituto Nacional de Vivienda y Urbanismo (INVU). (1954). Memoria Anual 1954. San José, Costa Rica.

Instituto Nacional de Vivienda y Urbanismo (INVU). (1985). Memoria Anual 1985. San José, Costa Rica.

Instituto Nacional de Vivienda y Urbanismo (Invu). (2001). Memoria Institucional 1998-2001. San José, Costa Rica.

Instituto Nacional de Vivienda y Urbanismo (INVU). (2002). Memoria Institucional 2002. San José, Costa Rica.

Kay, C. (2005). Enfoques sobre el Desarrollo Rural en América Latina y Europa desde Mediados del Siglo Veinte. Ponencia presentada en el Seminario Internacional Enfoque y perspectivas de la enseñanza del desarrollo rural. Bogotá, 1-2 de setiembre. Colombia, Pontificia Universidad Javeriana. 
León, J. (2012). Historia económica de Costa Rica en el siglo XX. Tomo II: la economía rural. San José, Costa Rica: IICE, CIHAC, UCR.

León, J.; Aguilar, J.; Chacón, M.; Peters, G.; Jara, A.; Villalobos, M. (2014). Crecimiento $y$ las políticas económicas. Tomo I: Historia económica de Costa Rica en el siglo $X X$. San José, Costa Rica: Editorial UCR, Universidad de Costa Rica.

Ministerio de Agricultura e Industria (MAI). (1952). Memoria Anual 1951. San José, Costa Rica.

Ministerio de Agricultura y Ganadería (MAG). (1963). Informe Anual de Labores 1962. San José, Costa Rica.

Ministerio de Agricultura y Ganadería (MAG). (1964). Informe Anual de Labores 1964. San José, Costa Rica.

Ministerio de Agricultura y Ganadería (MAG). (1967). Informe Anual de Labores 1966. San José, Costa Rica.

Ministerio de Educación Pública (MEP). (1950). Memoria Anual 1950. San José, Costa Rica.

Ministerio de Educación Pública (MEP). (1951). Memoria Anual 1951. San José, Costa Rica.

Ministerio de Educación Pública (MEP). (1963). Memoria Anual 1963. San José, Costa Rica.

Ministerio de Educación Pública (MEP).(1986). Memoria Anual 1982-1986. San José, Costa Rica.

Ministerio de Planificación Nacional y Política Económica (mideplan). (1982). Plan Nacional de Desarrollo 1982-1986, Volvamos a la Tierra, Tomos I y II. San José, Costa Rica.

Ministerio de Planificación Nacional y Política Económica (mideplan). (1987). Plan Nacional de Desarrollo 1986-1990, Tomos 1 y 2. San José, Costa Rica.

Ministerio de Planificación Nacional y Política Económica (mideplan). (1991). Plan Nacional de Desarrollo 1990-1994, Desarrollo Sostenible con Justicia Social. San José, Costa Rica.
Ministerio de Planificación Nacional y Política Económica (mideplan). (1994). Plan Nacional de Desarrollo 1994-1998 Francisco J Orlich. San José, Costa Rica.

Ministerio de Planificación Nacional y Política Económica (mideplan). (2001). Plan Nacional de Desarrollo Humano 1998-2002. Informe de Labores al tercer año de la Administración. San José, Costa Rica.

Ministerio de Planificación Nacional y Política Económica (Mideplan). (2003). Plan Nacional de Desarrollo Monseñor Víctor Manuel Sanabria 2002-2006. San José, Costa Rica.

Ministerio de Planificación Nacional y Política Económica (mideplan). (2007). Plan Nacional de Desarrollo José Manuel Dengo Obregón 2006-2010. San José, Costa Rica.

Ministerio de Planificación Nacional y Política Económica (mideplan). (2010). Plan Nacional de Desarrollo 2011-2014 María Teresa Obregón Zamora. San José, Costa Rica.

Ministerio de Transportes (мт). (1960). Informe Anual de Labores 1959-1960. Dirección General, MT, San José, Costa Rica.

Ministerio de Transportes (мт). (1966). Memoria Anual 1962-1966. Dirección General, MT, San José, Costa Rica.

Ministerio de Obras Públicas y Transportes (моРт). (1974). Memoria Anual 1973-1974. San José, Costa Rica.

Ministerio de Obras Públicas y Transportes (моРт). (1975). Memoria Anual 19741975. San José, Costa Rica.

Ministerio de Obras Públicas y Transportes (моРт). (1993). Memoria Anual 19921993. San José, Costa Rica.

Ministerio de Obras Públicas y Transportes (моРт). (2001). Memoria Anual 2000 2001. San José, Costa Rica.

Ministerio de Vivienda y Asentamientos Humanos (мIVAH). (2014). Memoria Anual 2002-2004. San José, Costa Rica.

Núñez, R. Marín, J.J. (2009). Acotando Espacios: Control estatal en Guanacaste 1860-1940: Construyendo el poder 
en Guanacaste. Guanacaste: (Re) Construcción de una región. 18502007. San José, Costa Rica: Librería Alma Mater.

Oficina de Planificación, Presidencia de la República (OPPR). (1966). Plan de Desarrollo Económico y Social de Costa Rica 1965-1968-1974. San José, Costa Rica.

Oficina de Planificación Nacional y Política Económica (OPNPE). (1979). Plan Nacional de Desarrollo 1979-1982. Gregorio José Ramírez. San José, Costa Rica.

Partido Liberación Nacional (PLN). (1973). Programa de Gobierno del Partido Liberación Nacional 1974-1978. San José, Costa Rica.

Programa Estado de la Nación (PEN). (2000). Sexto informe 1999 del Programa Estado de la Nación en Desarrollo Humano Sostenible. San José, Costa Rica.

Programa Estado de la Nación (PEN). (2013). Informe 2013 del Programa Estado de la Nación en Desarrollo Humano Sostenible. San José, Costa Rica.

Rovira, J. (1987). Costa Rica en los años 80. San José, Costa Rica: Edit Provenir.

Serrano, C. (1976). Historia de la Carretera Interamericana. (Tesis de Licenciatura en Historia). Facultad de Ciencias Sociales, Universidad de Costa Rica. San José, Costa Rica.

Viales, R. (2000). Los Liberales y la Colonización de las Áreas de Frontera no Cafetaleras: El Caso de la Región Atlántica (Caribe) Costarricense entre 1870 y 1930. (Tesis de Doctorado en Historia). Programa Ínteruniversitario de Doctorado en Historia Económica, Universitat Autónoma de Barcelona, España.

Viales, R. (2000a). Poblar, comunicar y buscar capitales: Tres fundamentos de la política agraria liberal en Costa Rica entre 1870 1930. Agronomía Costarricense, 24 (1). San José, Costa Rica: MAG.

Zeledón, F. (2009). La Construcción de la Carretera Inter-Americana en Guanacaste, región y medios de comunicación 1943 y 1970. Guanacaste: (Re) Construcción de una región. 1850-2007. San José, Costa Rica: Librería Alma Mater.

Fecha de ingreso: 17/05/2018 Fecha de aprobación: 11/10/2018 
